\title{
LOCALLY COMPACT HUGHES PLANES
}

\author{
MARKUS STROPPEL
}

\begin{abstract}
Among the eight-dimensional stable planes, the compact connected generalized Hughes planes and the geometries induced on the outer points are characterized by the property that these planes admit an effective action of the group $\mathrm{SL}_{3} \mathbb{C}$.
\end{abstract}

1. Introduction. In [15], H. Salzmann describes the compact connected (generalized) Hughes planes ( $c f$. [2]), and characterizes these planes among the eight-dimensional compact projective planes: These are the only planes of this type that admit a semi-simple (Lie) group $\Delta$ of automorphisms with $\operatorname{dim} \Delta \geq 16$. This semi-simple group is isomorphic with the almost simple group $\mathrm{SL}_{3} \mathbb{C}$, except in the desarguesian case (i.e., the plane over Hamilton's quaternions $\mathbb{W}$. This plane admits - in addition to $\mathrm{SL}_{3} \mathbb{C}$ - the thirty-fivedimensional simple group $\mathrm{PSL}_{3} \mathbb{H}$, the twenty-one-dimensional simple groups $\mathrm{PSU}_{3} \mathbb{H}(0)$ and $\operatorname{PSU}_{3} \mathbb{E}(1)$, and the semi-simple group Aut($(\mathbb{H}) \ltimes \mathrm{SL}_{2} \mathbb{H}$ of dimension 18, cf. [17]).

If $\mathbb{P}_{p}$ is a non-desarguesian compact connected Hughes plane then there is an invariant Baer subplane which is desarguesian (i.e., isomorphic with the projective plane over $\mathbb{C}$ ). The points and lines of this subplane are called inner, while all other elements of $\mathbb{P}_{f}$ are termed outer. The geometry induced on the set of outer points forms an eight-dimensional stable plane $\mathbb{M}_{\varphi}$ (in the sense of $R$. Löwen [12]), on which $\mathrm{SL}_{3} \mathbb{C}$ acts point-transitively with two line orbits (namely, inner and outer lines, respectively). The planes $\mathbb{P}_{p}$ and $\mathbb{M}_{p}$ will be defined precisely in the following chapter.

The present paper is devoted to the proof of the following result: Each eightdimensional stable plane $\mathbb{M}=(M, \mathcal{M})$ that admits $\mathrm{SL}_{3} \mathbb{C}$ as a group of automorphisms is isomorphic with $\mathbb{P}_{\varphi}$ or $\mathbb{M}_{\varphi}$ for some $\varphi$. This result contributes to a determination of the "most homogeneous" eight-dimensional stable planes, as begun in [17].

Of particular importance for our proof is the fact that, in contrast to the situation of the (finite) planes originally described by D. R. Hughes, the planes $\mathbb{P}_{f}$ and $\mathbb{M}_{f}$ admit the group $\mathrm{SL}_{3} \mathbb{C}$ rather than the simple group $\mathrm{PSL}_{3} \mathbb{C}$ : the set of fixed lines of the center $\mathrm{Z}=\left\{\varepsilon \mathbf{1} \mid \varepsilon^{3}=1\right\}$ shall give us a frame for the reconstruction of the geometry.

2. The planes. Let $\mathbb{P}=(P, P)$ be a compact connected generalized Hughes plane. According to [2] there is a ternary operation $T$ defined on some affine line $K$ of $\mathbb{P}$ such that the operations $x+y=T(1, x, y)$ and $x \circ y=T(x, y, 0)$ make $(K,+, \circ)$ a nearfield. Note, however, that $T$ is linear if, and only if, the plane $\mathbb{P}$ is desarguesian. Since $T$ can be described by geometric operations, the nearfield $(K,+, \circ)$ is a locally compact connected

Received by the editors June 2, 1992.

AMS subject classification: Primary: 51H10; secondary: 51H20, 51 A35.

(C) Canadian Mathematical Society 1994. 
nearfield. These have been classified by F. Kalscheuer ([8], $c f$. [22]); those that are not (skew) fields can be obtained as modifications of Hamilton's quaternions $\mathbb{H}$ :

RESULT 2.1. Let $\tilde{\varphi}: \mathbb{R}^{>} \rightarrow \mathbb{\mathbb { T }}$ be a continuous homomorphism, where $\mathbb{R}^{>}$is the multiplicative group of positive real numbers, and $\mathbb{T}$ is the multiplicative group of complex numbers of norm 1. For $x \in \mathbb{H}^{*}$ write $x^{\varphi}:=|x|^{\tilde{\varphi}}$. Then $H_{\varphi}=(\mathbb{H},+, 0)$ is a locally compact connected (left $\left.{ }^{1}\right)$ nearfield, where

$$
x \circ y:=\left\{\begin{array}{ll}
x x^{\bar{\varphi}} y x^{\varphi} & \text { if } x \neq 0 \\
0 & \text { if } x=0
\end{array} .\right.
$$

Except for the fields $\mathbb{R}$ and $\mathbb{C}$, each locally compact connected left nearfield is isomorphic with one of these ${ }^{2}$. Moreover, the nearfields $H_{\varphi}$ and $H_{\psi}$ are isomorphic if, and only if, $\varphi=\psi$ or $\varphi=\bar{\psi}$.

REMARKS 2.2. a) Note that the constant mapping $\varphi \equiv 1$ gives $H_{1}=\mathbb{H}$.

b) The following useful formulae are verified easily (recall that $j$ denotes a quaternion with $\bar{j}=-j, j^{2}=-1$ and $\mathbb{U}=\mathbb{C}+j \mathbb{C}$ ):

$$
\begin{gathered}
s \circ y=s y \quad \text { for } s, y \in \mathbb{U}, s \bar{s}=1 \\
x \circ c=x c \text { for each } c \in \mathbb{C} \\
j c=\bar{c} j \quad \text { for each } c \in \mathbb{C} \\
x \circ j \circ c=x \bar{c} \overline{x^{\varphi} j} \quad \text { for each } c \in \mathbb{C} \\
a \circ(x+y)=a \circ x+a \circ y \quad \text { but, in general, }(x+y) \circ a \neq x \circ a+y \circ a
\end{gathered}
$$

c) $\mathbb{C} \circ h+\mathbb{C}=\mathbb{C}+h \circ \mathbb{C}=\mathbb{U}$ for each $h \in \mathbb{U} \backslash \mathbb{C}(c f$. [2: p. 678]).

We shall now describe the compact connected Hughes planes. The set of points is

$$
P_{\varphi}=\left\{H_{\varphi} \circ(x, y, z) \mid(x, y, z) \in H_{\varphi}^{3} \backslash\{(0,0,0)\}\right\}
$$

on which a compact connected topology is induced by the canonical mapping

$$
H_{\varphi}^{3} \backslash\{(0,0,0)\} \rightarrow P_{\varphi}:(x, y, z) \longmapsto H_{\varphi} \circ(x, y, z) .
$$

In fact, the resulting space is an eight-dimensional compact connected manifold. Via right multiplication, the group $\mathrm{GL}_{3} \mathbb{C}$ of non-singular $3 \times 3$-matrices with entries in $\mathbb{C}$ acts on $H_{\varphi}^{3}$. This induces an action on $P_{\varphi}, c f$. Remarks 2.2. The kernel of this action is the one-parameter group $\left\{c \mathbf{1} \mid c \in \mathrm{C}_{\mathbb{C}^{*}}\left(H_{\varphi}\right)\right\}$, where $\mathrm{C}_{\mathbb{C}^{*}}\left(H_{\varphi}\right)$ denotes the centralizer of $H_{\varphi}$ in the multiplicative group $\mathbb{C}^{*}$. In particular, we infer that $\Delta=\mathrm{SL}_{3} \mathbb{C}$ acts effectively (faithfully).

The set $\mathcal{P}_{\varphi}$ of lines consists of the sets

$$
\left\{H_{\varphi} \circ(x, y, z) \mid(x a+y b+z c)+(x d+y e+z f) \circ s=0\right\}
$$

\footnotetext{
1 i.e., $a \circ(x+y)=a \circ x+a \circ y$.

2 of course, each right nearfield is anti-isomorphic with a left nearfield.
} 
where $a, b, c, d, e, f \in \mathbb{C}$ and $s \in \mathbb{H}$ such that the described set is non-empty and not equal to $P_{P}$. In this way, we obtain a projective plane $\mathbb{P}_{f}=\left(P_{f}, P_{f}\right)$, see [2]. Endowing $P_{F}$ with the topology induced from the Hausdorff metric derived from any metric on $P_{f}$ that induces the topology of $P_{P}$, one obtains that $\mathbb{P}_{\hat{p}}$ is a compact connected projective plane ([15]; applying [9], this result might also be derived by proving that the ternary operation

$$
T(x, s, t)= \begin{cases}x s+t & \text { if } s \in \mathbb{C} \\ (x+k) \circ s+k^{\prime} & \text { if } s \notin \mathbb{C} \text { and } t=k \circ s+k^{\prime} \text { with } k, k^{\prime} \in \mathbb{C}\end{cases}
$$

is continuous).

RESULT 2.3. According to [2:1.1], each line may be described by an equation of the form $^{3}$

$$
\begin{aligned}
& \text { i) } \quad y-z a=(x-z b) \circ m \quad \text { ii) } y=x a-z \circ \mathrm{s} \\
& \text { iii) } \quad x=z \circ s \quad \text { iv) } \quad z=0
\end{aligned}
$$

where $m \in H_{\varphi} \backslash \mathbb{C} ; a, b \in \mathbb{C}$ and $s \in H_{\varphi}$.

Both $\mathrm{GL}_{3} \mathbb{C}$ and $\mathrm{SL}_{3} \mathbb{C}$ act with two orbits on $P_{\mathrm{f}}$ and $\mathcal{P}_{f}$, respectively:

LEMMA 2.4. Under the action of $\Delta=\mathrm{SL}_{3} \mathbb{C}$, the orbit decompositions of the point and line space are

$$
P_{f}=p \Delta \cup q \Delta, \quad P_{f}=J \Delta \dot{\cup} I \Delta
$$

where $p=H_{\hat{r}} \circ(j, 1,0), q=H_{\hat{\gamma}} \circ(0,0,1), J$ is the outer line defined by $y=-x \circ j$, and $I$ is the inner line defined $b y z=0$.

Proof. We distinguish the cases as in Result 2.3. The line $I$ is the only one of type iv), while $J$ is of type i) with $a=0=b$ and $m=-j$. It is easy to see that, via matrices of the form $\left(\begin{array}{lll}0 & 1 & 0 \\ b & a & 1 \\ 1 & 0 & 0\end{array}\right),\left(\begin{array}{ccc}0 & 0 \\ 1 & a & 0 \\ 0 & 0 & -1\end{array}\right) \in \mathrm{SL}_{3} \mathbb{C}$, the lines of type i) and ii) can be moved to lines of type iii). If $s \in \mathbb{C}$ then these lines can obviously be moved to $I$. If $s=a+j b \notin \mathbb{C}$ (i.e., if $b \neq 0$ ) then equation iii) is equivalent to

$$
0=z \circ s-x=z \overline{z^{p}}(a+j b) z^{\hat{p}}-x=z a-x+(z \circ j) b .
$$

This line is also described by $0=z a b^{-1}-x b^{-1}+z \circ j$. Now $\left(\begin{array}{ccc}a & 0 & 1 \\ -b & 0 & 0 \\ 0 & -b & 1 \\ 0\end{array}\right) \in \Delta$ moves this line to $J$, and we have shown that $\mathcal{P}_{\tau}=J \Delta \cup I \Delta$.

The inner elements form a Baer subplane. For each point $w$ there is therefore an inner line $I \delta \in I \Delta$ such that $w \in I \delta$. Consequently, the point $w \delta^{-1}$ is of the form $w=H_{F} 0$ $(x, y, 0)$. If $y=0$, we may choose $x \in \mathbb{C}$ and obtain that $w$ is an inner point. Since $\Delta$ acts in the usual, transitive way on the set of inner points, we obtain that in this case $w=H_{f} \circ(1,0,0) \in q \Delta$. If $w$ is not an inner point, we may assume that $y=1$ and $x=a+j b$ with $a, b \in \mathbb{C}, b \neq 0$. Now $w\left(\begin{array}{cccc}b & 1 & 0 & 0 \\ b & 1 & a & 0 \\ 0 & 0 & b\end{array}\right)=H_{-} \circ(j, 1,0)=p$, and the assertion is proved.

\footnotetext{
3 Since we prefer vectors to be rows, with matrices acting from the right, we have to use left instead of right nearfields, and each of the formulate in $|2|$ is reversed.
} 
Lemma 2.5. Assume that $\Delta=\mathrm{SL}_{3} \mathbb{C}$ acts on $\mathbb{P}_{p}$ in the way described above. Then the stabilizers of $q, p, I, J$, respectively, are

$$
\begin{gathered}
\Delta_{q}=\Phi:=\left\{\left(\begin{array}{cc}
A & v^{\mathrm{t}} \\
& \frac{1}{\operatorname{det} A}
\end{array}\right) \mid A \in \mathrm{GL}_{2} \mathbb{C}, v \in \mathbb{C}\right\} \\
\Delta_{p}=\Lambda_{\varphi}:=\left\{\left(\begin{array}{cc}
A r r^{\varphi} & \\
v & \frac{1}{r r^{\varphi}}
\end{array}\right) \mid r \in \mathbb{R}^{>}, A \in \mathrm{SU}_{2} \mathbb{C}, v \in \mathbb{C}^{2}\right\} \leq \Phi^{\mathrm{t}} \\
\Delta_{l}=\Phi^{\mathrm{t}}=\left\{\left(\begin{array}{cc}
A & \\
v & \frac{1}{\operatorname{det} A}
\end{array}\right) \mid A \in \mathrm{GL}_{2} \mathbb{C}, v \in \mathbb{C}\right\} \\
\Delta_{J}=\Lambda_{\varphi}^{\mathrm{t}}=\left\{\left(\begin{array}{cc}
A r r^{\varphi} & v^{\mathrm{t}} \\
& \frac{1}{r r^{p}}
\end{array}\right) \mid r \in \mathbb{R}^{>}, A \in \mathrm{SU}_{2} \mathbb{C}, v \in \mathbb{C}^{2}\right\} \leq \boldsymbol{\Phi} .
\end{gathered}
$$

PROOF. i) We leave the computation of $\Delta_{q}$ and $\Delta_{I}$ to the reader, since this is well known.

ii) If $\delta \in \Delta_{p}$ then $(j, 1,0) \delta=(h \circ j, h, 0)$ for some $h \in \mathbb{H}^{*}$. Writing $\delta=\left(\begin{array}{c}x \\ D \\ y \\ y\end{array}\right)$ with $D \in \mathrm{GL}_{2} \mathbb{C}, v \in \mathbb{C}^{2}$ and $x, y, z \in \mathbb{C}$ we obtain that $j x+y=0$. Since $j$ and 1 form a basis of $\mathbb{t}$ over $\mathbb{C}$, we conclude that $x=y=0$ and $\delta \in \Phi^{\mathrm{t}}$. It remains to show that $D$ is of the form $A r r^{\varphi}$ with $A \in \mathrm{SU}_{2} \mathbb{C}$ and $r>0$.

iii) We may write $D=\left(\begin{array}{l}a b \\ c d\end{array}\right) r r^{\varphi}$ with $r>0$ and $b \bar{b}+d \bar{d}=1$. Then

$$
(j, 1) D=(j a+c, j b+d) r r^{p} \in H_{\varphi} \circ(j, 1)
$$

if, and only if,

$$
(j a+c) r r^{\imath}=(j b+d) r r^{\imath} \circ j
$$

which is equivalent to

$$
(j a+c) r^{\varphi}=(j b+d) r^{\varphi} \overline{r^{\hat{p}} j r^{\varphi}} .
$$

The right hand side equals $(j b+d) j r^{\varphi}$, and using Remarks 2.2 we obtain that $c=j b j=-\bar{b}$ and $d=-j a j=\bar{a}$. Thus $\left(\begin{array}{c}a b \\ -\bar{b} \bar{a}\end{array}\right) \in \mathrm{SU}_{2} \mathbb{C}$, and $\Delta_{p}=\Lambda_{\varphi}$.

iv) Similar calculations yield that $\Delta_{J}=\Lambda_{f}^{\mathrm{t}}$.

3. Some lemmas on actions of $\mathrm{SL}_{3} \mathbb{C}$. Before we turn to the study of actions of $\mathrm{SL}_{3} \mathbb{C}$ on eight-dimensional stable planes, we collect some general information.

LEMMA 3.1. The centralizer of $\mathrm{PSO}_{n} \mathbb{C}$ in $\mathrm{PSL}_{n} \mathbb{C}$ is trivial.

PROOF. For each non-isotropic vector $v \in \mathbb{C}^{n}$, there is a subgroup $\Xi$ of $\mathrm{SO}_{n} \mathbb{C}$ such that $\mathbb{C} v$ is exactly the subspace of fixed elements of $\Xi$. Therefore the centralizer of $\mathrm{PSO}_{n} \mathbb{C}$ acts trivially on the set of one-dimensional non-isotropic subspaces of $\mathbb{C}^{n}$. This set contains a frame for the $n-1$-dimensional projective space over $\mathbb{C}$, and the assertion follows. 
LEMMA 3.2. Assume that $\Delta=\mathrm{SL}_{3} \mathbb{C}$ acts almost ${ }^{4}$ effectively on a connected locally compact space $X$ of dimension $d \leq 4$. Then $X$ is homeomorphic with the complex projective plane, and the given action is equivalent to the usual one or its dual. In particular, the space $X$ is compact, the group $\Delta$ acts transitively with kernel $\mathrm{Z}=\mathrm{Z}(\Delta)$, and each stabilizer is a conjugate of $\Phi$ or $\Phi^{\mathrm{t}}$, respectively.

ProOF. i) According to [13], each orbit $x^{E}$ under the maximal compact subgroup $\mathrm{E}=\mathrm{SU}_{3} \mathbb{C}$ of $\Delta$ is either trivial or has full dimension. In the second case $x^{\mathrm{E}}$ is open and compact in $X$, and $x^{\mathrm{E}}=X$ since $X$ is connected. We infer that $\mathrm{E}$ (and, a fortiori, $\Delta$ ) acts transitively on $X$, and $X$ is compact. According to [13], the only possibility is that $X$ is homeomorphic with $\mathrm{P}_{2} \mathbb{C}$, and the action of $\mathrm{E}$ is equivalent to the usual one, i.e., each stabilizer $\mathrm{E}_{x}$ is a conjugate of $\Theta=\left\{\left({ }^{A}{ }_{\operatorname{det} A^{-1}}\right) \mid A \in \mathrm{U}_{2} \mathbb{C}\right\}$.

ii) It remains to determine the action of $\Delta$, i.e., to compare the stabilizer $\Delta_{x}$ with $\Phi$. In its usual action, the group $\Delta$ is 2-transitive, hence primitive on $P_{2} \mathbb{C}$. Therefore $\Phi$ is a maximal subgroup of $\Delta$. Since $\Phi$ is connected, it suffices to show that the connected component $\Gamma$ of $\Delta_{x}$ is a conjugate of $\Phi$. From the assumption that $\operatorname{dim} X=4$ we infer that $\operatorname{dim} \Gamma=12(=\operatorname{dim} \Phi)$.

iii) If $\Gamma$ acts irreducibly on $\mathbb{C}^{3}$ (the vector space underlying the complex projective plane), we obtain a decomposition $\Gamma=\Psi \Xi$, where $\Psi$ is the commutator subgroup and $\Xi$ is the identity component of the center of $\Gamma$ (see [3: $\S 6$, number 4, Proposition 5]). According to Schur's lemma, the centralizer of $\Gamma$ in $\mathrm{GL}_{3} \mathbb{C}$ is $\mathbb{C}^{*}$. We infer that $\Xi \leq$ $\mathrm{Z}\left(\mathrm{GL}_{3} \mathbb{C}\right) \cap \mathrm{SL}_{3} \mathbb{C}$. Connectedness implies that $\Xi=\mathbf{1}$, and $\Gamma$ is semi-simple.

iv) There is no almost simple group of dimension 12 . Since $\Gamma$ is a linear semi-simple group, each almost simple factor of $\Gamma$ contains a torus subgroup (i.e., a subgroup that is isomorphic with $\mathbb{T}=\mathrm{SO}_{2} \mathbb{R}$ ). Since $\Delta$ has rank 2 , there are exactly two almost simple factors of $\Gamma$. Each maximal compact subgroup of $\Gamma$ is a conjugate of $\Theta(c f . \mathrm{i})$ ). We infer that one of the almost simple factors of $\Gamma$ has a one-dimensional compact subgroup. This implies that the factors have dimension 3 and 9, respectively. This is impossible since there are no almost simple groups of dimension 9.

v) There remains the case where there exists a non trivial $\Gamma$-invariant subspace $U$ of $\mathbb{C}^{3}$. Then $\operatorname{dim}_{\mathbb{C}} U=1$ or 2 , and $\Gamma$ is a conjugate of a subgroup of $\Phi$ or $\Phi^{\mathrm{t}}$, respectively. The assertion follows from the fact that $\operatorname{dim} \Phi=\operatorname{dim} \Gamma$.

LEMMA 3.3. Let

$$
\begin{gathered}
\alpha=\left(\begin{array}{cc}
-1 & 0 \\
0 & 1
\end{array}\right), \quad Y=\mathrm{C}_{\Delta}(\alpha)=\left\{\left(\begin{array}{ll}
A & \\
\Omega & \operatorname{det} A^{-1}
\end{array}\right) \mid A \in \mathrm{GL}_{2} \mathbb{C}\right\} \text { and } \\
\\
=\left\{\left(\begin{array}{ll}
1 & 0 \\
v & 1
\end{array}\right) \mid v \in \mathbb{C}^{2}\right\} .
\end{gathered}
$$

Then $\Phi^{\mathrm{t}}=\Upsilon \Omega$, and an involution $\beta \in \Phi^{\mathrm{t}}$ belongs to the conjugacy class $\alpha^{\Omega}$ if, and only if, $\mathrm{C}_{\Delta}(\alpha) \leq \Phi^{\mathrm{t}}$.

\footnotetext{
4 i.e., the kernel is totally disconnected (in fact, finite).
} 
PROOF. In $\Delta$, the involutions form a single conjugacy class. Each involution $\beta \in \Delta$ induces a reflection on $\mathrm{P}_{2} \mathbb{C}$, and its centralizer $\mathrm{C}_{\Delta}(\beta)$ fixes center and axis, but no other point nor line. If $\mathrm{C}_{\Delta}(\beta) \leq \Phi^{\mathrm{t}}$ then $\mathrm{C}_{\Delta}(\beta)$ fixes the axis of $\alpha$. Therefore $\alpha$ and $\beta$ have the same axis. Each reflection on the complex plane is determined by its center and axis, and $\Phi^{t}$ acts transitively on the possible centers. Hence all reflections with the same axis as $\alpha$ are conjugates under $\Phi^{\mathrm{t}}$. From the fact that $\Phi^{\mathrm{t}}=\mathrm{C}_{\Delta}(\alpha) \Omega$ we infer that $\alpha^{\Phi^{\prime}}=\alpha^{\Omega}$.

4. Actions of $\mathrm{SL}_{3} \mathbb{C}$ on eight-dimensional stable planes. A stable plane is a linear space $\mathbb{M}=(M, \mathcal{M})$, where the point space $M$ and the line space $\mathcal{M}$ are endowed with locally compact Hausdorff topologies such that

- the geometric operations (joining points, intersecting lines) are continuous,

- the set of pairs of intersecting lines is open in $\mathcal{M} \times \mathcal{M}$ (axiom of stability).

Quite often, it is assumed in addition that the point space $M$ has positive and finite covering dimension. See [10] and [11] for general information.

Endowed with the compact-open topology derived from the action on the point space, the group Aut $(\mathbb{M})$ of all automorphisms (i.e., continuous collineations) of a stable plane $\mathrm{M}$ is a locally compact, separable transformation group. An action of a topological group $\Gamma$ on a stable plane $\mathbb{M}$ is a continuous homomorphism of $\Gamma$ to $\operatorname{Aut}(\mathbb{M})$. If the action is effective, we call $\Gamma$ a group of automorphisms of $\mathbb{M}$.

In the sequel, let $\mathbb{M}=(M, \mathcal{M})$ be an eight-dimensional stable plane, and assume that $\Delta=\mathrm{SL}_{3} \mathbb{C}$ is a group of automorphisms of $\mathbb{M}$. We use exponential notation for this given action. In this chapter, we shall assume that $M$ is connected, and that the center $\mathrm{Z}$ of $\Delta$ acts (fixed-point-)freely on $M$. The last chapter extends our result to arbitrary eight-dimensional stable planes (and arbitrary actions of $\Delta$ ).

\section{LEMMA 4.1. Each involution in $\Delta$ has an axis, but no center.}

PROOF. According to [21: $2.10 \mathrm{a}$ )], the eight-dimensional maximal compact subgroup $\mathrm{E}=\mathrm{SU}_{3} \mathbb{C}$ of $\Delta$ does not act transitively on $\mathbb{M}$. Hence $\operatorname{dim} \mathrm{E}_{x} \geq 1$ for each point $x \in M$, and $\mathrm{E}_{x}$ contains an involution. The involutions in $\Delta$ form a single conjugacy class, therefore each of them fixes at least one point. If an involution $\alpha \in \Delta$ has center $c$, then $c$ is fixed by Z. This contradicts our general assumption. If the set of fixed points of $\alpha$ carries a Baer subplane $\mathbb{F}$, then the centralizer $Y$ of $\alpha$ in $\Delta$ induces on $\mathbb{F}$ a group $\Psi$ of dimension 7 at least. The group $\Psi$ is the product of its commutator group $\Psi^{\prime} \cong \mathrm{PSL}_{2} \mathbb{C} \cong \mathrm{PSO}_{3} \mathbb{C}$ and its center, which has dimension $\geq 1$. According to [12], the four-dimensional plane $\mathbb{F}$ contains the complex oval plane, on which $\Psi^{\prime}$ acts in the usual way, and $\Psi$ embeds into PSL $_{3} \mathbb{C}$. By Lemma 3.1, this is impossible. There remains the case that each involution has an axis, but no center.

COROLLARY 4.2. The center $\mathrm{Z}$ of $\Delta$ acts quasi-perspectively (i.e., for each point $x \in M$ there is some line $L_{x}$ such that $x^{Z} \subseteq L_{x}$ ).

PROOF. Assume that there is an orbit $x^{Z}$ that is not contained in a line. Then each involution in $\Delta_{x}$ has a non-collinear set of fixed points (namely, $x^{Z}$ ), a contradiction. 
REMARK 4.3. Since we assume that $Z$ acts freely, this line $L_{x}$ is determined uniquely by $x$. The set $\mathcal{L}_{\mathrm{Z}}=\left\{L_{x} \mid x \in M\right\}$ consists of fixed lines of $\mathrm{Z}$, and it is locally homeomorphic with a line (cf. [20:1]).

COROLlary 4.4. Up to transposition, the following holds: For each point $p \in M$, the stabilizer $\Delta_{p}$ is a conjugate of a subgroup of $\Phi^{t}$.

Proof. According to Lemma 3.2, the four-dimensional connected set $\mathcal{L}_{\mathrm{Z}}$ is homeomorphic with $\mathrm{P}_{2} \mathbb{C}$, and $\Delta$ acts, up to transposition, in the usual way. The stabilizer $\Delta_{p}$ fixes the line $L_{p}$, and the stabilizer $\Delta_{L_{p}}$ is a conjugate of $\Phi^{\mathrm{t}}$.

LEMmA 4.5. Assume that $\Delta_{L_{p}}$ equals $\Phi^{\prime}$. Then the group $\langle\alpha\rangle \Omega$ acts trivially on $L_{p}$.

Proof. There is an involution $\beta \in \Delta_{p}$ that has some axis $A$. Since $Z$ leaves $A$ invariant and moves $p$, we conclude that $A=L_{p}$, and $\mathrm{C}_{\Delta} \beta \leq \Phi^{\mathrm{t}}$. Thus $\beta \in \alpha^{\Omega}$ by Lemma 3.3. The kernel of the action of $\Phi^{\mathrm{t}}$ on $L_{p}$ contains the conjugacy class $\alpha^{\Omega}$, hence all elements of the form $\alpha^{\omega} \alpha$, where $\omega \in \Omega$. Now the assertion follows from the fact that $\Omega=\{\alpha \omega \alpha \mid \omega \in \Omega\}$.

According to Lemma 4.5, each involution in $\Delta_{p}$ has axis $L_{p}$. From [20: Theorem 9a)] we infer:

COROLLARY 4.6. There are no commuting involutions in $\Delta_{p}$. In particular, the group $\Delta_{p}$ does not contain a subgroup that is isomorphic with $\mathbb{T}^{2}$ or $\mathrm{SO}_{3} \mathbb{R}$.

Lemma 4.7. Define $\mathrm{T}=\left\{\left(\begin{array}{lll}a & 0 & 0 \\ 0 & a & 0 \\ 0 & 0 & a^{2}\end{array}\right) \mid a \in \mathbb{C}, a \bar{a}=1\right\} \leq \Phi^{t}=\Delta_{L_{p}}$. For each point $q$ outside $L_{p}$, the orbits $p^{\mathrm{T}}$ and $q^{\mathrm{T}}$ generate a subplane of dimension 4 at least.

PROOF. The group $\mathrm{T}$ contains $\mathrm{Z}$, hence $\mathrm{T}$ moves each point, and each orbit is compact and one-dimensional. For each point $q$ outside $L_{p}$, the set $p^{\mathrm{T}} \cup q^{\mathrm{T}}$ generates therefore a subplane $\mathbb{E}$. If the lines of $\mathbb{E}$ are one-dimensional, then $p^{\mathrm{T}}$ is a compact line of $\mathbb{E}$ and meets each other line of $\mathbb{E}$ [10: 1.15]. In particular, the line $p^{\mathrm{T}}$ meets each other fixed line of $\mathrm{Z}$ in $\mathbb{E}$ in a point that is fixed by $\mathrm{Z}$ - a contradiction.

We shall need the following generalization of the compactness criterion [19: 6.3]:

LEMMA 4.8. Let $\mathbb{M}=(M, \mathcal{M})$ be an eight-dimensional stable plane. Assume that there is a locally compact group $\Lambda$ and a continuous monomorphism $\omega: \Lambda \rightarrow \operatorname{Aut}(\mathbb{M})$, i.e., an effective action of $\Lambda$ on $\mathbb{M}$. If with respect to this action a connected subgroup $\Theta$ of $\Lambda$ acts trivially on a Baer subplane $\mathbb{B}$ of $\mathbb{M}\left(\right.$ i.e., $\left.\Theta\right|_{\mathbb{B}}=1$ ) then the closure $\bar{\Theta}$ of $\Theta$ in Aut $(\mathbb{M})$ is a connected compact abelian group, and $\operatorname{dim} \Theta=\operatorname{dim} \bar{\Theta} \leq 1$. Consequently, the factor groups $\mathrm{N}_{\Lambda}(\Theta) / \mathrm{C}_{\Lambda}(\Theta)$ and $\mathrm{N}_{\text {Aut }(\mathrm{M})}(\bar{\Theta}) / \mathrm{C}_{\mathrm{Aut}(\mathrm{M})}(\bar{\Theta})$ are discrete.

PROOF. The closure of a connected set is connected again [6: V.1.6], and of course $\bar{\Theta}$ acts trivially on $\mathbb{B}$. From [19: 6.3] we infer that $\bar{\Theta}$ is compact abelian, and $\operatorname{dim} \bar{\Theta} \leq 1$. The action by conjugation is a continuous homomorphism $r: \mathrm{N}_{\mathrm{Aut}(\mathbb{M})}(\overline{\mathrm{\Theta}}) \rightarrow \operatorname{Aut}(\overline{\mathrm{\Theta}})$, where the latter is endowed with the $g$-topology [1]. Since $\operatorname{Aut}(\bar{\Theta})$ is topologically isomorphic with the automorphism group of the discrete dual of $\bar{\Theta}$, we infer that $\operatorname{Aut}(\bar{\Theta})$ and $\mathrm{N}_{\mathrm{Aut}(\mathrm{M})}(\bar{\Theta}) / \mathrm{C}_{\mathrm{Aut}(\mathrm{M})}(\bar{\Theta})$ are discrete. Now the assertion follows from the fact that $w$

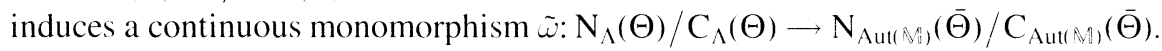


COROLLARY 4.9. If a connected subgroup $\Xi$ of $\Upsilon_{p}$ acts trivially on a subset $\mathcal{U}$ of $\mathcal{L}_{\mathrm{Z}}$ that accumulates at $L_{p}$, then $\operatorname{dim} \Xi \leq 1$, and $\mathrm{N}_{\Delta}(\Xi) / \mathrm{C}_{\Delta}(\Xi)$ is discrete.

Proof. The involution $\alpha$ fixes exactly two lines through $p$, namely the axis $L_{p}$ and some $C_{p}$. Since $\Xi \leq \mathcal{Y}=\mathrm{C}_{\Delta}(\alpha)$, both lines are fixed by $\Xi$. Choosing $L \in \mathcal{U}$ near $L_{p}$, we obtain that $L$ meets $C_{p}$ in some point $q$, and $\Xi$ fixes $q$. Since T centralizes $\Upsilon$, we conclude that $\Xi$ acts trivially on the orbits $p^{\mathrm{T}}$ and $q^{\mathrm{T}}$, and the assertion follows from Lemma 4.7.

LEMMA 4.10. a) The stabilizer $\Upsilon_{p}$ has a subgroup $\Sigma$ that is isomorphic with $\mathrm{SU}_{2} \mathbb{C}$.

b) The stabilizer $Y_{p}$ has no solvable subgroup of dimension 4.

c) The stabilizer $Y_{p}$ has no subgroup that is locally isomorphic with $\mathrm{SL}_{2} \mathbb{R}$.

Proof. i) Assume that $\Xi \leq \Upsilon_{p}$ is solvable, and that $\operatorname{dim} \Xi \geq 4$. Via conjugation, the group $\Xi$ acts $\mathbb{C}$-linearly on $\Omega \cong \mathbb{C}^{2}$. Since $\Xi$ is solvable, there is some $\omega \in \Omega \backslash \mathbf{1}$ such that $\omega^{\Xi}$ is contained in the $\mathbb{C}$-subspace spanned by $\omega$ (Lie's Theorem). We conclude that $\operatorname{dim} \mathrm{C}_{\Xi}(\omega) \geq 2$. Now $\mathrm{C}_{\Xi}(\omega)=\mathrm{C}_{\Xi}\left(\alpha^{\omega}\right)$ acts trivially on the set $\mathcal{U}$ consisting of the axes of the elements $\alpha^{\omega^{\prime}}$, where $\omega^{\prime}$ runs over the $\mathbb{C}$-subspace spanned by $\omega$. Thus we have obtained a contradiction to Corollary 4.9 , proving b).

ii) Assume that $\Psi \leq Y_{p}$ is locally isomorphic with $\mathrm{SL}_{2} \mathbb{R}$. Since the $\mathbb{C}$-linear action of $Y$ on $\Omega$ (via conjugation) is almost effective, there is no proper $\Psi$-invariant $\mathbb{C}$-subspace of $\Omega$. Up to equivalence, the universal covering of $\mathrm{SL}_{2} \mathbb{R}$ has only one irreducible complex representation of degree 2 . We conclude that, in $Y$, the group $\Psi$ is a conjugate of $\left\{\left(\begin{array}{c}{ }^{A} \\ \\ 1\end{array}\right) \mid A \in \mathrm{SL}_{2} \mathbb{R}\right\}$. Hence we may assume that $\Gamma=\left\{\left(\begin{array}{lll}1 & b & 0 \\ 0 & 1 & 0 \\ 0 & 0 & 1\end{array}\right) \mid b \in \mathbb{R}\right\}$ is contained in $\Psi$. But $\Gamma$ fixes a connected set of lines in $\mathcal{L}_{Z}$ that contains $L_{p}$. Again, we obtain a contradiction to Corollary 4.9 , and c) is proved.

iii) We have that $\operatorname{dim} Y_{p} \geq \operatorname{dim} Y-4=4$. According to $b$ ), the identity component of $Y_{p}$ is not solvable. Therefore it contains an almost simple subgroup of dimension 3. Each such group is locally isomorphic with $\mathrm{SU}_{2} \mathbb{C}$ or $\mathrm{SL}_{2} \mathbb{R}$. Now assertion a) follows from Corollary 4.6 and c).

LEMMA 4.11. The group $\Omega$ acts regularly on $\mathcal{M}_{p} \backslash\left\{L_{p}\right\}$.

ProOF. i) Let $L \neq L_{p}$ be a line through $p$, and let $\xi \in \Omega_{L}$. Since $Y$ acts transitively on the set of one-dimensional subspaces of $\Omega \cong \mathbb{C}^{2}$, we may assume that $\xi=\left(\begin{array}{lll}1 & 0 & 0 \\ 0 & 1 & 0 \\ x & 0 & 1\end{array}\right)$ for some $x \in \mathbb{C}$. Then $C_{Y}(\xi)=\left\{\left(\begin{array}{ccc}a & 0 & 0 \\ b & a^{-2} & 0 \\ 0 & 0 & a\end{array}\right) \mid a \in \mathbb{C}^{*}, b \in \mathbb{C}\right\}$. Because $\Psi=C_{Y}(\xi)$ is solvable and four-dimensional, the point $p$ is moved by $\Psi$, see Lemma $4.10 \mathrm{~b}$. The element $\xi$ acts trivially on the line orbit $L^{\Psi \Omega}$, and there are $\psi \in \Psi$ and $\omega \in \Omega$ such that the lines $L$ and $L^{l \omega}$ meet outside $L_{p}$. The intersection point is fixed by $\xi$ but moved by $\Omega$, hence $\xi$ fixes more than one point outside $L_{p}$. Since $L_{p}$ is the axis of $\xi$, we conclude that $\xi=\mathbf{1}$. Hence $\Omega$ acts semi-regularly on $\mathcal{M}_{p} \backslash\left\{L_{p}\right\}$. 
ii) For each line $L \in \mathcal{M}_{p} \backslash\left\{L_{p}\right\}$, the orbit $L^{\Omega}$ has dimension 4. According to [12:11 c)], the orbit is open. Connectedness of $\mathcal{M}_{p} \backslash\left\{L_{p}\right\}$ (cf. [10:1.14, 1.19], [12:11 a)]) implies that $L^{\Omega}=\mathcal{M}_{p} \backslash\left\{L_{p}\right\}$, and $\Omega$ acts regularly.

COROLLARY 4.12. The reconstruction method of [18] applies to $\Delta$ and $\mathbb{M}_{\vec{r}}$.

ProOF. The group $\Delta$ acts transitively on $M_{p}$ and has two orbits on $\mathcal{M}_{p}$. From Lemma 4.11 we infer that the stabilizer $\Delta_{p}$ has two orbits in $\mathcal{M}_{p}$ (namely, the trivial orbit $\left\{L_{p}\right\}$ and its complement).

Lemma 4.13. Assume that $\Delta_{L_{p}}=\Phi^{\prime}$. Then there is a homomorphism $\varphi: \mathbb{R}^{>} \longrightarrow \mathbb{C}^{*}$ such that $\Delta_{p}$ is a conjugate of $\Lambda_{\vec{r}}$.

ProOF. i) Let $\Sigma$ be a subgroup of $\Upsilon_{p}$ that is isomorphic with $\mathrm{SU}_{2} \mathbb{C}$ ( $c$ f . Lemma 4.10a). Being almost simple, this subgroup is contained in the commutator subgroup of $Y$, which is isomorphic with $\mathrm{SL}_{2} \mathbb{C}$. Each subgroup of $\mathrm{SL}_{2} \mathbb{C}$ that is isomorphic with $\mathrm{SU}_{2} \mathbb{C}$ is a maximal compact subgroup, hence these subgroups form a single conjugacy class of maximal subgroups. In order to determine $Y_{p}$, we study the projection $i$ of $Y$ to $Y / Z(Y)$ : The image $Y_{p}^{n}$ equals $\Sigma^{r}$, and $Y_{p}=\mathrm{K} \Sigma$, where $\mathrm{K}=\mathrm{Y}_{p} \cap Z(Y)$ is the kernel of the restriction of $\kappa$ to $Y_{p}$.

ii) According to Corollary 4.6, there is no torus subgroup in $\mathrm{K}$, hence $\operatorname{dim} \mathrm{K} \leq 1$ and $\operatorname{dim} Y_{p} \leq 4$. This implies that $\operatorname{dim} p^{Y}=4$, and $p^{Y}$ is open in $L_{p}$. Since $\mathrm{K}$ commutes with $Y$, we obtain that $\mathrm{K}$ acts trivially on $p^{\mathrm{Y}}$.

iii) From Richardson's characterization [14] of the actions of compact groups on the four-sphere we infer that each element of $\Sigma$ fixes exactly two lines through $p$ : namely, the axis $L_{p}$ of $\alpha$ and the line $C_{p}$ (cf. [20: 7]). The group K has the semi-axis ${ }^{5} L_{p} \supseteq p^{\Upsilon}$, therefore each element of prime power order in $\mathrm{K}$ has axis $L_{p}$ and fixes exactly one line $L$ in $\mathcal{M}_{p} \backslash\left\{L_{p}\right\}$. Since $\mathrm{K}$ commutes with $\alpha$, we infer that $L=C_{p}$.

iv) If $\mathrm{K} \cap \mathrm{T}$ is not contained in $\Sigma$, then there is a non-cyclic finite abelian subgroup of $\mathrm{K} \Sigma$. Consequently, the group $\mathrm{K} \Sigma$ contains a non-cyclic elementary abelian subgroup A. According to iii), this group fixes $L_{p}$ and $C_{p}$ and acts semi-regularly on $\mathcal{M}_{p} \backslash\left\{L_{p}, C_{p}\right\}$. By a theorem of P. Smith [16] this implies that the cohomology of A is cyclic, which means that A is cyclic [4: Chapter XII, $\S 11$, Theorem 11.6]-a contradiction.

v) We have shown that $\mathrm{K} \cap \mathrm{T}=\mathrm{K} \cap \Sigma=\langle\alpha\rangle$. Therefore $\mathrm{K}$ is the direct product of its compact-free identity component and $\langle\alpha\rangle$. This means there is a homomorphism $\varphi: \mathbb{R}^{>} \rightarrow \mathrm{Z}(Y) \cong \mathbb{C}^{*}$ such that $\mathbb{R}^{*}$ equals the identity component of K. Now $\Delta_{p}=$ $\Upsilon_{p} \Omega=\Lambda_{f}$, and the assertion is proved.

COROLLARY 4.14. The stabilizer $\Delta_{p, C_{p}}$ equals $\mathrm{Y}_{p}$.

PROOF. We have that $\Upsilon_{p}$ fixes $C_{p}$ because $\alpha$ commutes with $Y$. On the other hand $\Delta_{p}=\Upsilon_{p} \Omega$ and $\Omega_{C_{p}}=\mathbf{1}$ by Lemma 4.11 .

5 A semi-axis of $K$ is a line that contains an open set of fixed points of $K$. 
LEMMA 4.15. Let $p$ be a point such that $\Delta_{p}=\Lambda_{\varphi}$. Then $\Delta_{C_{p}}=\Lambda_{\varphi}^{\mathrm{t}}$.

PROOF. From the fact that $\operatorname{dim} \Delta_{C_{p}} / \Delta_{p, C_{p}} \leq 4$ we infer that $\operatorname{dim} \Delta_{C_{p}}=8$. Moreover, we know that $\Delta_{C_{p}}$ contains $\Delta_{p, C_{p}}=\mathrm{Y}_{p} \cong \mathbb{R} \times \mathrm{SU}_{2} \mathbb{C}$.

i) Each semi-simple group of dimension 8 is locally isomorphic with $\mathrm{SU}_{3} \mathbb{C}, \mathrm{SU}_{3} \mathbb{C}(1)$, or $\mathrm{SL}_{3} \mathbb{R}$. The only group of this type that contains a closed subgroup that is isomorphic with $\Upsilon_{p}$ is the universal covering of $\mathrm{SU}_{3} \mathbb{C}(1)$, which is not a linear group. Therefore $\Delta_{C}$, is not semi-simple.

ii) Via the adjoint representation, the reductive group $Y_{p}$ acts completely reducibly on the Lie algebra $\llbracket$ of $\Delta_{C_{p}}$. Effective linear actions of the commutator subgroup $\Sigma=$ $\left\{\left({ }^{A}{ }_{1}\right) \mid A \in \mathrm{SU}_{2} \mathbb{C}\right\}$ are at least four-dimensional. Since $\operatorname{dim}_{\Delta_{C_{p}}}(\Sigma) \leq \operatorname{dim}_{\Delta}(\Sigma)=$ 2, we infer that $\mathfrak{l}$ decomposes into a direct $\operatorname{sum} \mathfrak{l}=\mathfrak{u} \oplus \mathfrak{v}$, where $\mathfrak{u}$ is the Lie algebra of $\Upsilon_{p}$ and $\mathfrak{v}$ is a four-dimensional vector space on which $\Sigma$ acts effectively and irreducibly. This implies that $\Sigma$ is a Levi complement in the identity component $\Gamma$ of $\Delta_{C_{p}}$ (recall that there are no semi-simple groups of dimension 7). Consequently, the radical $\mathrm{P}$ of $\Gamma$ is a five-dimensional characteristic subgroup of $\Delta_{C_{p}}$. In its natural action on $\mathbb{C}^{3}$, the solvable group $\mathrm{P}$ leaves invariant a flag $0<U<V<\mathbb{C}^{3}$.

iii) We consider the vector space $W$ that is generated by the $\Delta_{C_{p}}$-images of $U$ in $\mathbb{C}^{3}$. If $W=\mathbb{C}^{3}$, then the five-dimensional group $\mathrm{P}$ is a conjugate of a subgroup of $\left\{\left(\begin{array}{ccc}a & 0 & 0 \\ 0 & b & 0 \\ 0 & 0 & (a b)^{-1}\end{array}\right) \mid a, b \in \mathbb{C}^{*}\right\}$, which is four-dimensional. This is impossible. Therefore $W$ is a proper subspace of $\mathbb{C}^{3}$. Since $\Sigma$ leaves $W$ invariant, we conclude that $W$ represents axis or center of $\alpha$ (in the action on $\mathrm{P}_{2} \mathbb{C}$ ). In the first case $\Delta_{C_{p}} \leq \Phi^{\mathrm{t}}=\Delta_{L_{p}}$, and we conclude that $\Delta_{C_{p}}=\Delta_{p, C_{p}}$, a contradiction. There remains the case where $\Delta_{C_{p}} \leq \Phi$.

iv) Consider the canonical mapping $\kappa: \Phi \rightarrow \Upsilon \cong \Phi / \Omega^{\mathrm{t}}$. The kernel $\Omega^{\mathrm{t}} \cap \Delta_{C_{p}}$ of the restriction of $\kappa$ to $\Delta_{C_{p}}$ is an $Y$-invariant subgroup of $\Omega^{\mathrm{t}}$, hence it is trivial or equals $\Omega^{\mathrm{t}}$. In the first case, we infer from the equation $\operatorname{dim} \Delta_{C_{p}}=8=\operatorname{dim} \Upsilon$ that $\Delta_{C_{p}}$ is isomorphic with $Y$, in contradiction to ii). Hence $\Omega^{t} \leq \Delta_{C_{p}}$, and we conclude that the identity component of $\Delta_{C_{p}}$ equals $Y_{p} \Omega^{\mathrm{t}}$. For each $\delta \in \Delta_{C_{p}}$, there are $\omega \in \Omega^{\mathrm{t}}$ and $v \in \mathrm{Y}$ such that $\delta=\omega v$ (recall that $\Delta_{C_{p}} \leq \Phi=\Omega^{\mathrm{t}} \Upsilon$ ). Now $v=\omega^{-1} \delta \in \Upsilon_{C_{p}}=\Upsilon_{p}$, and the assertion follows.

Combining Corollary 4.12, Lemma 4.13, and Lemma 4.15 we obtain

PROPOSITION 4.16. If $\Delta=\mathrm{SL}_{3} \mathbb{C}$ acts on a connected eight-dimensional stable plane $\mathbb{M}$ such that the center $\mathrm{Z}$ of $\Delta$ acts freely, then there is some homomorphism $\varphi: \mathbb{R}^{>} \rightarrow \mathbb{C}^{*}$ such that $\mathbb{M}$ is isomorphic with $\mathbb{M}_{\varphi}$. Such an action is always obtained from an arbitrary effective action of $\Delta$ on an eight-dimensional stable plane if one restricts the action of $\Delta$ to the subplane induced on an orbit $p^{\Delta}$, where $p$ is a point that is not fixed by $\mathrm{Z}$.

5. The general case. The following is the key to the extension of Proposition 4.16 to arbitrary eight-dimensional stable planes:

LEMMA 5.1. Let $\mathbb{M}=(M, \mathcal{M})$ be a stable plane, and let $E$ be an open subset of $M$. Denote by $\mathcal{E}$ the set of lines that meet $E$ in more than one point. For each point $x \in E$ 
and each line $L \in \mathcal{E}$ that does not pass through $x$, write $\|_{L . x}^{E}$ for the set of lines through $x$ that do not meet $L \cap E$. If $E$ is a proper subset of $M$ then there is a point $x \in E$ and a line $L \in \mathcal{E}$ such that

a) the set $\|_{L, x}^{E}$ is non-empty. In particular, the geometry $(E, \mathcal{E})$ is not a projective plane.

b) If the complement $M \backslash E$ has non-empty interior then the set $\|_{L . x}^{E}$ has non-empty interior.

Proof. Choose points $a \in E$ and $b \in M \backslash E$. Then there is a point $x \in E \backslash a b$, and $x b \in \|_{L x}^{E}$. If there is a neighbourhood $U$ of $b$ in $M$ that does not meet $E$, then the set $\{x u \mid u \in U \cap a b\}$ forms a neighbourhood in $\|_{L, x}^{E}$.

THEOREM 5.2. If $\Delta=\mathrm{SL}_{3} \mathbb{C}$ acts effectively on an eight-dimensional stable plane $\mathbb{M}=(M, \mathcal{M})$, then there is a homomorphism $\varphi: \mathbb{R}^{>} \rightarrow \mathbb{C}^{*}$ such that $\mathbb{M}$ is isomorphic with $\mathbb{P}_{\varphi}$ or $\mathbb{M}_{\varphi}$ (depending on whether or not there exist fixed points of $\mathrm{Z}$ ), and the action of $\Delta$ is equivalent to the usual one or its dual.

Proof. According to Proposition 4.16, there is an open point orbit $p^{\Delta}$ such that the subplane induced on $p^{\Delta}$ is isomorphic with $\mathbb{M}_{\varphi}$ for some $\varphi$. Now Lemma $5.1 \mathrm{~b}$ shows that the complement of $p^{\Delta}$ has empty interior. Therefore each point in $M \backslash p^{\Delta}$ is fixed by $\mathrm{Z}$ (cf. Proposition 4.16). Applying [21:2.10 c)] to $\mathrm{E}=\mathrm{SU}_{3} \mathbb{C}$, we obtain that the set $F$ of fixed points of $\mathrm{Z}$ is not contained in a line. Therefore $\mathrm{Z}$ acts trivially on the subplane $\mathbb{F}=(F, \mathcal{F})$ that is induced on $F$. From $[21: 2.10$ a) $]$ we infer that $\mathbb{F}$ is isomorphic with the projective plane over $\mathbb{C}$, and the action of $E$ is equivalent to the usual one. In particular, $\Delta$ acts transitively on $F$. For $z \in F$ the set $z M=\left\{z m \mid m \in p^{\Delta}\right\}$ is open in $\mathcal{M}_{-}$. Therefore $z M \cap C_{p}^{\Delta}$ is not empty. For each point $x \in p^{\Delta} \backslash C_{p}$ the set $\|_{C_{p}, x}^{p^{\wedge}}$ consists just of one line. Hence $C_{p}$, as a line of $\mathbb{M}$, is projective (i.e., it meets any other line in $\mathcal{M}$ ). On the other hand, any two inner lines intersect in the projective plane $\mathbb{F}$. Thus each pair of lines has a common point in $\mathbb{M}$, and $\mathbb{M}$ is projective. Since $F=M \backslash p^{\Delta}$ has empty interior, the subplane that is induced on $p^{\Delta}$ is dense and determines $\mathbb{M} \cong \mathbb{P}_{f}$.

\section{REFERENCES}

1. R. Arens, Topologies for homeomorphism groups, Amer. J. Math. 68(1946), 593-610.

2. M. Biliotti, Su una generalizzazione di Dembowski dei piani di Hughes, Boll. Un. Mat. Ital. B (5) 16(1979), 674-693.

3. N. Bourbaki, Groupes et algèbres de Lie, Chapter I, Hermann, Paris, 1971.

4. H. Cartan and S. Eilenberg, Homological algebra, Princeton Univ. Press, 1956.

5. P. Dembowski, Generalized Hughes planes, Canad. J. Math. 23(1971), 481-494.

6. J. Dugundji, Topology, Allyn \& Bacon, Boston, 1966.

7. D. R. Hughes, A class of non-desarguesian projective planes, Canad. J. Math. 9(1957), 378-388.

8. F. Kalscheuer, Die Bestimmung aller stetigen Fastkörper über dem Körper der reellen Zahlen als Grundkörper, Abh. Math. Sem. Univ. Hamburg, 13(1940), 413-435.

9. N. Knarr and C. Weigand, Ein Kriterium für topologische Ternärkörper, Arch. Math. 46(1986), 368-370.

10. R. Löwen, Vierdimensionale stabile Ebenen, Geom. Dedicata 5(1976), 239-294.

11. Topology and dimension of stable planes: On a conjecture by H. Freudenthal, J. Reine Angew. Math. 343(1983), 108-122. 
12. _ Actions of $\mathrm{SO}(3)$ on 4-dimensional stable planes, Aequationes Math. 30(1986), 212-222.

13. L. N. Mann, Dimensions of compact transformation groups, Michigan Math. J. 14(1967),433-444.

14. R. Richardson, Groups acting on the 4-sphere, Illinois J. Math. 5(1961), 474-485.

15. H. Salzmann, Kompakte, 8-dimensionale projektive Ebenen mit großer Kollineationsgruppe, Math. Z. 176(1981), 345-357.

16. P. A. Smith, New results and old problems in finite transformation groups, Bull. Amer. Math. Soc. 66(1960), $401-415$.

17. M. Stroppel, Achtdimensionale stabile Ebenen mit quasieinfacher Automorphismengruppe, Dissertation, Tübingen, 1991.

18. Reconstruction of incidence geometries from groups of automorphisms, Arch. Math. 58(1992), $621-624$

19. _ _ Planar groups of automorphisms of stable planes, J. Geom. 44(1992), 184-200.

20. _ Quasiperspectivities in stable planes, Monatsh. Math. 115(1993), 183-189.

21. Compact groups of automorphisms of stable planes, Forum Math. (1993), to appear.

22. J. Tits, Sur les groupes doublement transitifs continus: correction et compléments, Comm. Math. Helv. 30(1956), 234-240.

Fachbereich Mathematik

Technische Hochschule Darmstadt

Schloßgartenstr. 7

D-64289 Darmstadt

Germany

e-mail:stroppel@mathematik.th-darmstadt.de 